\title{
Desarrollo y globalización neoliberal en territorios indígenas de la Huasteca hidalguense, México
}

\author{
Development and neoliberal globalization in indigenous territories of the Huasteca hidalguense, \\ Mexico \\ Jorge Dolores Bautista ${ }^{a}$
}

\begin{abstract}
:
From a geographical perspective, this article analyses the effect of the confluence of regional development processes with neoliberal globalization in the transformation of indigenous territories. Around these issues establishes a theoretical and interdisciplinary discussion on the scale effect produced by globalization in the territory, debating the idea homogenization to give way to the study of the peculiarities derived from interactions Social, political and cultural. As empirical reference there take the indigenous territories nahuas located in the microbasin of the Rio Venado, municipality of Atlapexco, Hidalgo State.
\end{abstract}

\section{Keywords:}

Regional development, neoliberal globalization, geography, landscape, indigenous territories

\section{Resumen:}

Desde una perspectiva geográfica, el presente artículo analiza el efecto de la confluencia de los procesos de desarrollo regional con la globalización neoliberal en la transformación de los territorios indígenas. En torno a estos temas establece una discusión teórica e interdisciplinaria sobre el efecto de escala que produce la globalización en el territorio, debatiendo la idea homogeneización para dar paso al estudio de las particularidades derivadas de las interacciones sociales, políticas y culturales. Como referencia empírica se toman los territorios indígenas nahuas localizados en la microcuenca del Río Venado, municipio de Atlapexco, estado de Hidalgo.

\section{Palabras Clave:}

Desarrollo, globalización neoliberal, geografía, paisaje, territorios indígenas

Artículo recibido: 1 de agosto de 2019

Dictaminado: 3 de septiembre de 2019

Segunda versión: 24 de octubre de 2019

Aceptado: 26 de octubre de 2019

${ }^{\text {a }}$ Universidad Autónoma del Estado de Hidalgo, Profesor de Tiempo Completo del Instituto de Ciencias Sociales y Humanidades. Área Académica de Trabajo Social. https://orcid.org/0000-0002-5076-8451, Email: jorge_dolores@uaeh.edu.mx 


\section{Introducción}

La tarea de la geografía se concibe como la de establecer un sistema crítico que abarque la fenomenología del paisaje, con el propósito de aprehender en todo sus significados y color la variedad de la escena terrestre.

Carl O. Sauer (2006)

La Huasteca es una región que se localiza en el norte del estado Hidalgo, identificable por las diversidades ecosistémicas y culturales. Sus paisajes se caracterizan por la confluencia fisiográfica que tienen las partes bajas de la Sierra Madre Oriental con las llanuras costeras del Golfo de México. Socioculturalmente, estos paisajes están construidos por las prácticas de la cosmovisión nahua que se manifiestan en la agricultura, la medicina tradicional y el uso de la lengua originaria como vehículo comunicante. En contraste, también tienen gran vitalidad las prácticas de una cultura mestiza dedicada fundamentalmente a la ganadería, que en el pasado reciente ejerció sobre la población local un férreo control económico y político.

El paisaje regional también muestra las huellas del desenlace de la lucha agraria que enfrentó, en los años setenta y ochenta, a decenas de comunidades indígenas en contra de los terratenientes e integrantes de la élite local que subordinaron a la Huasteca durante la mayor parte del siglo XXi. Este período es el antecedente inmediato del arribo de la dimensión neoliberal de la globalización a estos territorios, donde se enmarcan las transformaciones contemporáneas de las relaciones sociales, las prácticas culturales y el medio ambiente (Dolores, 2015).

Estas transformaciones requieren ser estudiadas en función de la particularidad de sus cambios, lo que implica el reto de generar nuevo conocimiento y soluciones a la complejidad de problemas que están aconteciendo en los territorios indígenas. Como respuesta a ese reto, desde la Geografía, se han incorporado de manera interdisciplinaria, referentes conceptuales como espacio, región, paisaje, territorio, lugar y frontera, que han sido útiles para el estudio de la complejidad de los fenómenos sociales que están transformando los territorios indígenas.

Una de las áreas donde ese reto manifiesta especial interés, se localiza en el análisis de los efectos que el desarrollo económico nacional y la globalización neoliberal han generado en las regiones indígenas. En distintos momentos históricos ambos procesos han generado transformaciones políticas, nuevas dinámicas territoriales y su particular realización local (desarrollo y globalización) conforme a la interacción con los elementos de las realidades socioculturales.

De acuerdo con lo anterior, es necesario plantear algunas interrogantes: ¿cómo estudiar de manera adecuada los territorios que componen una región sin perder de vista los vínculos de interrelación con otras escalas del espacio?; ¿es el territorio una escala adecuada de análisis para comprender los cambios que acontecen al interior de las regiones?; ¿cómo analizar las transformaciones regionales con los cambios que a nivel nacional y global están sucediendo actualmente?

Para responder estas preguntas, en el presente texto, primero me abocaré a analizar los efectos que ha tenido el desarrollo regional; posteriormente, analizaré el papel de la globalización neoliberal en la reelaboración del territorio indígena, análisis que haré desde las transformaciones que ha experimentado el territorio de las comunidades nahuas, localizadas en la microcuenca del Río Venado, municipio de Atlapexco, Hidalgo".

El análisis da importancia a los cambios del paisaje, considerando que es una forma adecuada para comprender los cambios producidos por un proyecto de desarrollo regional implementando durante los años ochenta, como medida para desactivar un movimiento social y cuya continuidad hubiera tenido consecuencias imprevistas. Este movimiento se organizó en torno a la añeja demanda por hacer efectivas resoluciones presidenciales que convertían en ejidatarios o comuneros a campesinos indígenas de esta región. Sin embargo, esas resoluciones no fueron efectivas, debido al uso de estrategias legales y las formas caciquiles de ejercicio del poder por parte de terratenientes, que se habían apropiado de las mejores tierras de los 
campesinos para dedicarlas a la ganadería y la agricultura comercial.

Posterior a la fase de modernización (principio de los años noventa), la región fue incluida en los esquemas de políticas asistencialistas, otorgados a regiones marginadas. Esta acción fue implementada, luego de la transformación neoliberal del Estado mexicano, como medida de compensación social para la mayor parte de las regiones indígenas y rurales del país, período en el que se enmarca la dinámica contemporánea de transformación territorial de este trabajo.

De acuerdo con lo planteado hasta aquí, la estrategia metodológica fue de carácter cualitativa y consistente en la realización de períodos de trabajo abocados a la elaboración de lecturas de paisaje, entrevistas a profundidad, registro audiovisual y observación participante. Esto permitió construir datos que fueron contrastados con información documental y análisis de tipo geográfico sobre los efectos sociales, políticos y culturales, resultantes de la inclusión regional en los esquemas de desarrollo de las primeras etapas del neoliberalismo mexicano.

Conforme a lo planteado, se sugiere que los cambios territoriales son multidimensionales y de escala diversa, pero que se vinculan a los procesos de globalización neoliberal en forma discontinua y acorde a las características socioculturales de la región o territorio. Se finaliza con un énfasis que coloca a los territorios indígenas como espacios de vitalidad política y sociocultural, donde día a día se están construyendo algunas de las respuestas más trascendentes a los efectos del desarrollo y de la globalización neoliberal.

\section{La confluencia del desarrollo como estrategia de crecimiento económico y las primeras manifestaciones del neoliberalismo en México}

Durante la década de los años setenta comenzó a tomar forma un fenómeno económico conocido como internacionalización del capital (Barkin, 1978), que estuvo caracterizado por el impulso que corporaciones empresariales dieron a la expansión del libre comercio.
Este proceso fue alentado por la presión que estas corporaciones hicieron a sus respectivos gobiernos, con la finalidad de lograr que a nivel internacional los intercambios comerciales fluyeran con menores restricciones arancelarias.

En ese contexto, algunas vertientes de pensamiento geográfico describieron que ese cambio implicaba, además de la menor regulación del comercio mundial, la profunda reconfiguración de la funcionalidad de las regiones periféricasiii. Esta reconfiguración generó nuevos canales de vinculación regional al mercado mundial, ocasionando que las nuevas funcionalidades incorporaran a los ámbitos de la vida cotidiana un sinnúmero de nuevos hábitos culturales, alimenticios, tecnológicos y productivos originados por su inédita incorporación, según sea el caso, a los procesos de producción en masa y el consumo de productos industrializados.

Las regiones, algunas ya incorporadas de manera temprana y protagónica a los procesos de reproducción de capital, experimentaron procesos de transformación ocasionados por las nuevas dinámicas de producción. En el ámbito rural, algunas regiones que habían estado al margen de los esquemas de desarrollo económico, fueron incorporadas al mercado; lo que sucedió como consecuencia de la expansión de las fronteras agrícolas y por la creación de nuevas zonas de producción agroindustrial.

Lo anterior también se dio por la configuración de nuevos polos de desarrollo, cuyo espacio social fue estructurado en torno a la producción de insumos necesarios para lo que comenzaba a perfilarse como los nuevos parámetros de producción industrial deslocalizada (Delgado, 2003). Esos procesos de reconfiguración trastocaron la faz del mundo y en países con cierto grado de desarrollo económico como México, ocasionaron la profundización de la desigualdad regional entre aquellas regiones que desde tiempo atrás ya estaban insertas dentro de los flujos de intercambio comercial, con las que comenzaban a ser objeto de algún tipo de proyecto desarrollo económico (RIMISP, 2018). ${ }^{\text {iv }}$ 
El alcance de esta reconfiguración llegó hasta las denominadas "regiones de refugio" (Aguirre Beltrán, 2009), espacios locales que habían estado al margen de los proyectos de modernización y desarrollo económico.

A esas regiones, la modernidad económica hizo su arribo en la forma de esquemas de desarrollo regional que fueron implementados con el objetivo de generar crecimiento económico para superar las condiciones de pobreza.

Esa perspectiva de desarrollo generó diversos cambios, que poco tiempo después, tomarían una nueva dimensión vinculada a la expansión de la globalización neoliberal, reorientándose hacia la ocupación de nuevas zonas para el abasto de materias primas y recursos energéticos. Para los territorios indígenas, esa dinámica, además de haberlos incorporado al mercado, modificó también los ámbitos de la vida cotidiana, y las dinámicas territoriales manifestaron el surgimiento de nuevos actores sociales, así como la diversificación de los procesos socioeconómicos.

En ese sentido, los cambios que las regiones indígenas están experimentando son el resultado de la combinación de factores locales y globales, lo que delinea un reto importante para analizar de manera particularizada el modo en que esos procesos están transformando la construcción del territorio. Una forma adecuada de estudiar esos cambios, proviene de la construcción analítica que vincula el paisaje, el territorio y el espacio social desde una perspectiva geográfica que aporta al estudio multidisciplinario de los cambios regionales y territoriales.

\section{Reproducción del neoliberalismo y globalización}

Para el pensamiento geográfico identificado con las corrientes constructivistas (Hiernaux y Lindón, 2006), el espacio social es concebido como una totalidad social y subjetivamente elaborada, conformada por la acción significante de seres humanos reunidos a través del tiempo en una diversidad de culturas y sociedades, considerando al espacio como una analogía del mundo donde se desenvuelve existencia individual y las acciones colectivas.

Sin embargo, el espacio social no es un referente exclusivo del pensamiento geográfico, ya que ciencias como la sociología y la filosofía, son coincidentes al considerar al espacio social como una totalidad social producida por las relaciones sociales y de producción, la apropiación territorial del mismo y su significación.

Para el sociólogo francés, Pierre Bourdieu (1999), el espacio social es una interacción simbólica entre actores sociales diferenciados por campos sociales, habitus y capitales sociales. Por su parte, para el sociólogo inglés, Anthony Giddens (2011), el espacio social es una estructuración de tiempo y espacio, donde las personas son agentes sociales que interactúan en una red de regionalizaciones donde la espacialidad toma forma concreta en las delimitaciones geográficas, culturales y políticas, productos de los posicionamientos sociales que los actores construyen para confrontar la vida cotidiana.

En contraste, para el filósofo francés, Henri Lefebvre (1976), el espacio social es una totalidad fragmentada donde el capitalismo neoliberal se reproduce a través de relaciones de producción dirigidas verticalmente y cuyo significado simbólico se encuentra en las trazas de las morfologías que produce en el territorio. En esta visión, el espacio es una totalidad social, cuya percepción es fragmentada como consecuencia de las barreras ideológicas que los grupos sociales construyen con base a prácticas de territorialidad.

El pensamiento de Lefevbre explica que la fragmentación del espacio social refleja su grado de instrumentalización, es decir su caracterización como objeto de reproducción capitalista. Enfatiza que así como se fragmenta el espacio, lo mismo sucede con las percepciones del espacio, teniendo como consecuencia la parcelación del conocimiento de la realidad socialv ${ }^{v}$. Estas modificaciones resignifican el sentido del espacio, haciéndolo mucho más particularizado y delimitado, convirtiéndolo en un instrumento de desarrollo de una nueva fase capitalista. De este modo, la compraventa de porciones de espacio representa una de las más visibles 
formas de instrumentación, en el sentido de que "El hecho de vivir no sólo se reduce a una función asignable, aislable y localizable" (Lefebvre, 1976:09) sino a una función que enmarca la vida social en el ámbito de la mercantilización del hábitat y del espacio público.

Este argumento esclarece que el espacio no se fragmenta de manera espontánea, sino de modo planificado y controlado por agentes que actúan de acuerdo a propósitos específicos e ideologías. En esta dinámica se localizan claves que ayudan a descifrar el papel que desempeñan aquellos agentes que tienen la capacidad para dirigir los procesos de transformación del espacio, lo que significa ejercicio de territorialidad y conocimiento de los objetivos, aspiraciones e ideologías que justifican la instrumentalización del espacio.

El espacio también está conformado por dimensiones simbólicas, como la del espacio vivido y las representaciones mentales del mismo, que guardan una estrecha relación con respecto a su producción. Pero el espacio, además de reproducirse en las relaciones sociales, también se disputa y por lo tanto es político.

El carácter político del espacio refiere la "definición" que le asignan sus usuarios a través de tres dimensiones de planificación: la material, la funcional y la del espaciotemporal. Mediante esta diferenciación, puede entenderse la manera en que el neoliberalismo transformó los esquemas de acumulación capitalista. El espacio fue convertido en objeto de consumo, generando procesos de disputa política entre actores sociales que intentan apropiarlo para un fin concreto $y$ en contra de aquellos que se les oponen o que buscan un modo distinto de integración; la afirmación de Lefebvre al respecto refiere que "el espacio es política" (1976:52).

En el marco del neoliberalismo, donde el Estado ha reducido sus ámbitos de participación, existen agentes dominantes que tienen la capacidad de congeniar los desacuerdos sociales en torno a la reproducción de los intereses económicos con las aspiraciones sociales. Esto puede observarse en la realización de algunas de las conocidas como "obras de infraestructura", que al idealizar progreso, dinamizan los procesos de reproducción de intereses privados que demuestran que "el espacio se expande y se produce por el desarrollo de las fuerzas de producción" (Lefebvre, 2008:78). La contradicción y el conflicto en el espacio se manifiestan en la centralización de los nodos de poder, así como su influencia y dominio territorial. A esto podríamos denominarlo como "descentralización centralizada", ya que la deslocalización mantiene las ubicuidades del poder que mantienen el control de los flujos mercantilistas que establecen dominio. Esta caracterización de las dinámicas capitalistas neoliberales permite ocupar nuevos espacios, lo que aunado a la creciente disponibilidad de dispositivos tecnológicos de información, como dice Castells (2002), ha contribuido a anular o disminuir distancias y fronteras que obstaculizaban la reproducción de capital, característica fundamental para la expansión de la globalización neoliberal.

En ese sentido, las regiones se han convertido en espacios que al ser reconfigurados a través de funciones o especializaciones productivas, contribuyen a la reproducción de intereses económicos. Esta característica es histórica y se remonta a los procesos de ocupación de nuevos territorios encabezados por países que ante la expansión del capitalismo, tuvieron que ocupar espacios más allá de sus fronteras para poder continuar con la reproducción de capital. Ese proceso en la globalización se ha acelerado, pero tiene integrado rasgos históricos que anticipan que la elaboración del espacio nunca ha sido neutra ni objetiva.

\section{Resignificación del espacio social y transformación del territorio indígena}

Conocer las condiciones en que se producen las relaciones sociales, quién las dirige y con qué propósito, son la principal crítica de Lefebvre al estructuralismo marxista, ya que descarta que el capitalismo se sostenga puramente en los aparatos ideológicos del Estado o por la vía política. Para él son relaciones de dominio diversas las que mantienen la instrumentalización del espacio, por lo que el 
capitalismo es reinventado de manera constante, como sucede en el neoliberalismo a través de la fragmentación e instrumentalización del espacio, tal como ha sucedido con la resignificación de los símbolos del progreso.

En el marco de la globalización neoliberal, la resignificación muestra un modo particular de instrumentalización del espacio que refleja el fracaso o el éxito del progreso según el prisma con el que se mire. Esto tiene la característica común de haber hecho visibles prácticas políticas que han contribuido a la reproducción del proyecto de las clases dominantes, que de manera localizada permite observar la transformación neoliberal del Estado, la modificación de las interacciones territoriales, los cambios en la estructura socioproductiva y la transformación de la morfología del paisaje como una constatación visible de la transformación del espacio en un momento determinado de su historia.

Al respecto, como se dijo al principio, los conceptos de espacio, región, territorio, lugar, frontera, son términos que han sumado al estudio complejo de los cambios que están transformando el mundo y su efecto que tienen en el entendimiento, reelaboración, reconstitución y fragmentación de los territorios indígenas.

Para el caso de las comunidades nahuas de la Huasteca hidalguense, asentadas en la microcuenca del Río Venado, los cambios que refieren la transformación de su paisaje, muestran las huellas de la llegada del desarrollo regional como respuesta a la inconformidad social que amenazó en convertirse en subversión armada y las de la funcionalización regional otorgada por el Estado neoliberal a través de la instrumentación de una política social de corte asistencialista.

El paisaje de ese territorio muestra de modo tangible y no abstracto la transformación social, refiriendo en el territorio rasgos más precisos de la relación de esos cambios con los que en otras latitudes transformaron otros territorios indígenas y otras regiones culturales o económicas del mundo. Para este trabajo son relevantes, desde una perspectiva integral, los cambios que en el espacio social de los territorios indígenas generan los planes de desarrollo regional.

Lo anterior puede entenderse a partir de lo siguiente: Hace casi 40 años, en una de las últimas políticas gubernamentales del Estado mexicano revolucionario, se buscó lograr de nueva cuenta la autosuficiencia alimentaria, creando para ello el ambicioso Sistema Alimentario Mexicano (SAM). Este sistema tenía como objetivo la modernización agroindustrial de diversas regiones rurales mexicanas para integrarlas en la dinámica de desarrollo, de aquel momento, como espacios productores de alimentos destinados al consumo nacional.

En la Huasteca hidalguense se desarrolló un programa piloto del SAM que fue conocido como Plan Integrado de Habilitación de la Huasteca Hidalguense (PIHHH) (Ruvalcaba, 1990). Este plan tenía como eje principal la creación de un distrito de riego, la conformación de unidades productivas de trabajo, la capacitación técnica, así como, la modernización de la infraestructura de caminos y de salud. Sin embargo, ante los vaivenes financieros y políticos, acontecidos a inicios de los años ochenta, el proyecto fue abandonado e irremediablemente vino el fracaso de su principal objetivo: el distrito de riego no se consumó.

No obstante este fracaso, sucedieron cambios notables: debido a la apertura de caminos se acabó con el aislamiento geográfico, se incrementó el tendido eléctrico, se abrieron más escuelas y la población indígena tuvo la gran posibilidad de reconstituir sus territorios como consecuencia de las expropiaciones de tierras, salida que el gobierno estatal y federal dieron a la confrontación entre campesinos y terratenientes. El resultado de esos cambios conforma el actual paisaje de estos territorios indígenas y expresa el significado de la transformación de su morfología, mostrando también que los cambios de la vida cotidiana no están desligados del dominio, de los cambios políticos y de los cambios globales.

Por tanto, en el paisaje del territorio de estas comunidades nahuas pueden observarse los efectos de las territorialidades ejercidas desde hace 40 años, 
cuando en la Huasteca hidalguense las poblaciones indígenas iniciaron una tenaz lucha para recuperar sus territorios.

\section{Geografía, procesos complejos de cambio social y construcción de los territorios indígenas}

Desde la perspectiva descrita, es necesario intensificar el análisis de enfoques integrales que construyan nuevo conocimiento sobre los cambios locales, pero que como en el caso de los territorios indígenas, al mismo tiempo vinculen el conocimiento de esos cambios con las transformaciones que están aconteciendo a nivel global. En este tenor, se han dado pasos al pasar de la visión indigenista sobre el estudio de los pueblos originarios, para dar cabida a perspectivas que permitan estudiar los espacios sociales indígenas no como territorios aislados, sino como lo que son: territorios dinámicos construidos por tendencias sociales diversas, que muestran gran vitalidad para conformar sus propios posicionamientos sociales, alternativas, disyuntivas y paradojas ante los efectos de la globalización.

En un plano similar, la apertura de la geografía a los procesos sociales no sólo en el plano del espacio geográfico, permitió generar nuevas escalas de análisis como la del territorio, el lugar y el espacio vivido para manifestar que en los referentes de particularidad espacialmente localizada se produce la diversidad social.

Esta forma de análisis nos remonta a la obra de Sauer (2006), para quien es importante analizar la capacidad humana del uso social del espacio, fuerza transformadora de las distintas culturas para usar los recursos naturales, lo que muestra modos diferenciados de aprendizaje, adaptación y aprovechamiento de los recursos del medio ambiente para subsistir. Para él, la distinción de esa diferenciación es significativa para establecer parámetros de la importancia que tiene la diversidad social.

En ese sentido, el pensamiento geográfico como representación analítica del mundo, no es ajeno a los cambios sociales. Una muestra de esto puede observarse en el resurgimiento, luego de años de dominio cuantitivista, del pensamiento crítico y humanista en la geografía (Sauer, 2003); tendencia proveniente de la complejidad de los eventos que la humanidad vive durante los años sesenta y setenta en el marco de la Guerra Fría y las contradicciones del modelo de crecimiento económico.

Don Mitchell (2000), geógrafo norteamericano, refiere que los acontecimientos de esas décadas ocasionaron un cisma en los campos de la ciencia social, permitiendo la posibilidad de incorporar de manera firme la diversidad como categoría analítica. Esta permitió relacionar el surgimiento de nuevas prácticas sociales vinculadas a particularidades culturales, históricas, étnicas, de género y clase social, donde los actores se incorporaron a apropiaciones del espacio ya establecidas o para la conformación de nuevas territorialidades.

Específicamente, ese cambio en la geografía y en otras ciencias sociales se le denominó como un giro cultural caracterizado por el renovado interés en el estudio del marxismo, la fenomenología y el humanismo. Es decir, en la crítica a la modernidad y la globalización como reflejo de integración económica subordinada, nuevas geografías encontraron nuevos elementos de análisis.

Una de ellas, por ejemplo, se relaciona con la importancia que tiene el modo en que es representado el mundo para actuarlo, como refiere David Lowenthal (2008), quien al tomar en cuenta la percepción social de los modos de comportamiento en el espacio, analiza la estructura social, la cultura y el lenguaje en la elaboración de lo que define como "geografías personales", donde interactúan los aspectos cognitivos del ser humano con relación a su apropiación espacial.

Para estas geografías, los actores sociales al posicionarse ante los procesos de cambio, refieren la importancia que tiene la percepción de los seres humanos con sus espacios de vida. Acorde a esto, las relaciones sociales configuran la racionalidad material del espacio, pero también exhiben su grado de subjetividad con relación a la influencia de las 
percepciones y significados que los sujetos establecen con sus entornos.

\section{Conclusiones}

Conforme a lo dicho hasta aquí, ahora corresponde volver a las preguntas iniciales. Una región no puede ser estudiada como un espacio homogéneo, requiere reconocer las delimitaciones territoriales que interactúan en su interior y por supuesto, establecer vínculos de análisis regional, nacional y global.

La globalización neoliberal ha logrado incrementar el comercio y las transacciones comerciales durante las últimas tres décadas. Una de las estrategias para lograr este objetivo, ha consistido en la reconfiguración de los espacios regionales. Esta reconfiguración ha significado la transformación de antiguas áreas de producción industrial o agropecuaria, así como la ocupación de nuevos espacios para la producción de mercancías que demanda el mercado y para la extracción de materias primas, lo que ha tenido repercusiones en las poblaciones que habitan tales espacios.

Algunos esquemas de análisis abordan estos efectos a partir del estudio del proceso específico de reconversión del espacio regional, dando poca importancia al resto de procesos que vinculan un mecanismo de reconfiguración con cambios que enlazan lo local, regional, nacional y global. Este tipo de procesos son los que hacen del territorio una escala de análisis adecuada para comprender el tipo específico de transformaciones locales, lo que responde a la segunda pregunta de este texto.

De acuerdo con esto, hay tareas pendientes para conocer el efecto que en la Huasteca han tenido procesos posteriores al desarrollo y la globalización, como son la migración de jornaleros hacia las zonas de producción agroindustrial, localizadas principalmente en el norte de México, la reactivación de la vocación ganadera, el papel de la actividad turística y el extractivismo.

Los efectos ocasionados por el neoliberalismo en las poblaciones indígenas se remontan a los años ochenta, cuando México se vuelve signatario del GATT ${ }^{\text {vii }}$, acuerdo clave para los futuros procesos de neoliberalización económica que transformaron los estados latinoamericanos. Paradójicamente, esa apertura en el espacio analizado coincide con la implementación del último esfuerzo por parte del Estado mexicano para devolver la soberanía alimentaria al país, el Sistema Alimentario Mexicano.

Desde entonces, las poblaciones nahuas de la Huasteca hidalguense han reajustado sus condiciones de vida ante diversos esquemas de desarrollo regional. El argumento de que la región es pobre, a pesar de las millonarias sumas que para política social se transfieren hacia esa región, es la justificación con la que los diferentes niveles de gobierno han recreado diversos esquemas de política asistencialista. Ante esta realidad, las poblaciones han construido respuestas al desarrollo y al neoliberalismo, dando nuevos elementos de análisis para los efectos que la globalización ejerce en sus espacios de vida, tal como sucede con la migración hacia las zonas agroindustriales mencionadas.

Parece ser que a pesar del reparto de tierra, la construcción de caminos, la transferencia de recursos y otros aspectos de la modernidad como las clínicas rurales, la electricidad y la educación no han sido suficientes para detener el flujo comunitario. El análisis de los efectos locales de la globalización neoliberal, del desarrollo como estrategia de crecimiento económico y la migración, son tres factores importantes para tener una nueva perspectiva de los efectos del neoliberalismo en los territorios indígenas.

Geográficamente, esto vincula distintas escalas del espacio que al ser transformadas por los procesos de reconfiguración neoliberal, experimentan cambios en su vida cotidiana, crean nuevos flujos de relación socioeconómica entre regiones diversas y plantean nuevos elementos para el análisis de los efectos de la reconfiguración neoliberal de los espacios regionales. Esto es una dimensión de análisis multidimensional que empírica y teóricamente vincula diferentes situaciones que forman parte de un mismo fenómeno, la globalización neoliberal y su dimensión geográfica. 
Finalmente, la respuesta a la tercera pregunta está relacionada con la construcción de escalas interrelacionadas de análisis, para analizar la heterogeneidad social. Las regiones son delimitaciones que indican diversidades culturales, económicas y políticas. Sin embargo, el territorio demuestra que al interior de las regiones existen diversidades que muestran modos de pertenencia social y apropiamiento del espacio que son complejos, históricos, simbólicos y contradictorios.

Generar nuevo conocimiento, soluciones participativas, horizontales y colaborativas a las problemáticas de los territorios, es uno de los más grandes retos de los científicos sociales. Para ello, el nuevo conocimiento sobre los territorios indígenas requiere trascender el nivel de análisis para dar el paso a la elaboración, en conjunto con las poblaciones indígenas, de estrategias abocadas al acotamiento de la desigualdad, la preservación de los recursos naturales y los ámbitos de libertad para que las poblaciones locales decidan su propio destino.

Estas estrategias también deben estar abocadas a garantizar los derechos humanos a un medio ambiente sano, al agua y al saneamiento, a la lengua local y a la no discriminación. Acciones en este sentido, serán en beneficio no sólo de los pueblos indígenas, ya que tendrán impactos positivos en el resto de la sociedad.

Para los gobiernos de las entidades federativas del país con territorios indígenas esto es condición necesaria para llevar a los hechos acciones de bienestar para la población. Para el caso de Hidalgo, la diversidad de pueblos indígenas que coexisten en el estado tiene que ser un factor a incorporar en la generación de políticas públicas interculturales.

Si bien los problemas contemporáneos tienen un mismo punto de partida, la solución a ellos es más compleja y al mismo tiempo sencilla. Por muchas causas, para los gobiernos ha sido complejo consultar y tomar en cuenta a los habitantes de los territorios. Pero si se fomentan las formas de dialogo horizontal, se camina hacia la sencillez, se identificará que muchas de las soluciones a los problemas ya existen. No en balde a la población indígena también se les conoce como la gente de los ecosistemas (Dasman, 1964 citado en Boege, 2008:23), gracias a que sus conocimientos y forma de utilizar los recursos naturales, a través del tiempo, les ha permitido coexistir con el medio ambiente.

\section{Referencias Bibliográficas}

Aguirre Beltrán, Gonzalo. (2009). Regiones de refugio: el desarrollo de la comunidad y el proceso dominical en mestizo de América. México: Universidad Veracruzana, Xalapa.

Barkin, David. (1978).Desarrollo regional y reorganización campesina. La Chontalpa como reflejo del problema agropecuario mexicano. Centro del Ecodesarrollo- México: Editorial Nueva Imagen,.

Boege, Eckart. (2008). "El patrimonio biocultural de los pueblos indígenas de México. Hacia la conservación in situ de la biodiversidad y agrodiversidad en los territorios indígenas". , México: INAH-CDI.

Bourdieu, Pierre. (1999). La distinción. Criterios y bases sociales del gusto. México. Taurus.

Castells, Manuel. (2002). La era de la información. La Sociedad Red Vol. 1. México, Distrito Federal: Siglo XXI Editores.

Delgado Mahecha, Ovidio. (2003). Debates sobre el espacio en la Geografía contemporánea. Bogotá: Universidad Nacional de Colombia-Red de Estudios de Espacio y Territorio.

Dolores Bautista, Jorge. (2015). Transformación de la geografía social de la Huasteca hidalguense; en Territorios, seguridad y soberanía alimentaria, Plata Vázquez, Fuensanta Medina y Ávila Méndez (coords.), pp. 173-203. México: El Colegio de San Luis.

Giddens, Anthony. (2011). La Constitución de la Sociedad. Bases para la Teoría de la Estructuración. México: Amorrortu Editores.

Harvey, David. (2007). Breve Historia del Neoliberalismo. Madrid, España: Akal..

Hiernaux, D; Alicia Lindón (2006). Tratado de Geografía Humana., , México: Anthropos/ UAM Iztapalapa.

Lefebvre, Henri. (1976). Espacio y política. El derecho a la ciudad II. Barcelona: Ediciones Península.

Lefebvre, Henri. (2008). "The Production of Space". USA: Blackwell. MA.

Lowenthal, David. (2008). A la découverte des paisajes de prédilection, en Pasajes du temps sur le paisaje. Infolio, Collection Archigraphy Témoignages. Pp.17-68

Mitchell, Don (2000). Cultural Geography. London: Blackwell.

RIMISP. (2018). Pobreza y Desigualdad, Informe Latinoamericano 2018. No dejar a ningún territorio atrás. Santiago de Chile: RIMISP-Centro Latinoamericano para el Desarrollo Rural.

Ruvalcaba Mercado, Jesús. (1990). El Plan Huasteca: sus causas y consecuencias, en La Huasteca: vida y milagros. México: CIESAS.

Sauer, Carl. (2006). La morfología del paisaje. Polis, Revista de la Universidad Bolivariana, vol. 5, núm. 15, 2006. Chile: Universidad de Los Lagos. Santiago. 


\title{
Notas
}

\begin{abstract}
i Aún persiste la desconfianza mutua entre mestizos y comunidades, haciéndose presente el racismo y renovadas formas de clientelismo y faccionalismo. En la región se viven los efectos del fracaso del proyecto de desarrollo regional, denominado Distrito de Riego Huasteca Hidalguense, el cual fue diseñado para dar solución a los conflictos agrarios. Actualmente están en desarrollo nuevos procesos de reconfiguración regional en torno al turismo, la reactivación de la vocación ganadera y la probable extracción de petróleo.
\end{abstract}

ii Los datos empíricos corresponden a períodos de trabajo de campo realizados en la región de estudio entre los años 2013-2015. Éstos fueron realizados como parte de la fase de recopilación de información para la elaboración de la tesis doctoral "El desarrollo regional en un paisaje de la Huasteca hidalguense: modernización, territorialidades y neoliberalismo, 1976-2012."

iii Por regiones periféricas se denomina a aquellas que hasta la segunda mitad del siglo XX estuvieron al margen de los procesos de desarrollo nacional, específicamente regiones rurales o indígenas.

iv El análisis de la desigualdad territorial, es uno de los que componen las áreas más importantes para entender el grado diferenciado de desarrollo que se manifiesta al interior de los estados nacionales. A escala local establece matices que permiten entender que el valor de los indicadores de desarrollo nacional no es homogéneo en todas las regiones que integran un país.

${ }^{v}$ Una de las premisas de esta teoría consiste en considerar al espacio como la representación holística de la realidad social.

${ }^{v i}$ Esta reflexión influenció profundamente el pensamiento de geógrafos como David Harvey, donde el primero enfatiza el uso de conocimientos, técnicas las denomina él, para la producción del espacio; y el segundo, en el señalamiento de que las regiones son soluciones espaciales que el capitalismo ha creado en beneficio de sus intereses.

${ }^{v i i}$ El Acuerdo General sobre Comercio y Aranceles fue el antecedente directo previo a la creación de la Organización Mundial de Comercio. 